\title{
$Z_{2}\left(Z_{2}+u Z_{2}\right)\left(Z_{2}+u Z_{2}+u^{2} Z_{2}\right)$-Additive Cyclic Codes
}

\section{Zhihui Li}

Department of Mathematics, School of Mathematics and Statistics, Shandong University of Technology, Zibo, China

\section{Email address:}

li_zhihui1@163.com

\section{To cite this article:}

Zhihui Li. $\mathrm{Z}_{2}\left(\mathrm{Z}_{2}+\mathrm{uZ} \mathrm{Z}_{2}\right)\left(\mathrm{Z}_{2}+\mathrm{uZ} \mathrm{Z}_{2}+\mathrm{u}^{2} \mathrm{Z}_{2}\right)$-Additive Cyclic Codes. Engineering Mathematics. Vol. 3, No. 2, 2019, pp. 30-39. doi: 10.11648/j.engmath.20190302.11

Received: June 14, 2019; Accepted: October 11, 2019; Published: October 25, 2019

\begin{abstract}
In this paper, we introduce the algebraic structure of $Z_{2}\left(Z_{2}+u Z_{2}\right)\left(Z_{2}+u Z_{2}+u^{2} Z_{2}\right)$-additive codes and $Z_{2}\left(Z_{2}+u Z_{2}\right)$ $\left(Z_{2}+u Z_{2}+u^{2} Z_{2}\right)$-additive cyclic codes. Compared to the $Z_{2} Z_{4} Z_{8}$-additive codes, the Gray image of any $Z_{2}\left(Z_{2}+u Z_{2}\right)$ $\left(Z_{2}+u Z_{2}+u^{2} Z_{2}\right)$-linear code will always be a linear binary code. Therefore, we consider the $Z_{2}\left(Z_{2}+u Z_{2}\right)\left(Z_{2}+u Z_{2}+u^{2} Z_{2}\right)-$ additive cyclic codes as a $\left(Z_{2}+u Z_{2}+u^{2} Z_{2}\right)$ [x] -submodule of $Z_{2}^{\alpha} \times\left(Z_{2}+u Z_{2}\right)^{\beta} \times\left(Z_{2}+u Z_{2}+u^{2} Z_{2}\right)^{\theta}$. We give the definition of $Z_{2}$ $\left(Z_{2}+u Z_{2}\right)\left(Z_{2}+u Z_{2}+u^{2} Z_{2}\right)$-additive codes with generator matrices and parity-check matrices. Furthermore, we give the fundamental result on considering their additive cyclic codes with generator polynomials and spanning sets.
\end{abstract}

Keywords: Additive Codes, Cyclic Codes, Minimal Generating Set

\section{Introduction}

Codes over rings were introduced in early 1970s. However this research topic had been widely and really concerned after a milestone paper written by Hammons et al. in 1994, which shown that some special classes of non-linear binary codes could be obtained as Gray images of linear codes over $Z_{4}$ [1]. Since then, several families of codes over finite rings have been studied by many scientists [2-4].

In 1973, Delsarte defined additive codes in terms of association schemes as the subgroups of the underlying abelian group [5]. In 2010, Borges et al. brought a new perspective to additive codes over rings, introducing Z2Z4additive codes [6]. Under binary Hamming scheme, the underlying group of order $2^{k}$ isomorphic to $Z_{2}{ }^{\alpha} \times Z_{4}{ }^{\beta}$, where $\alpha$ and $\beta$ are nonnegative integers. The subgroups of underlying group are called $Z_{2} Z_{4}$-additive codes. Therefore, a $Z_{2} Z_{4}$-additive code $C$ is defined to be a subgroup of $Z_{2}{ }^{\alpha} \times Z_{4}{ }^{\beta}$, where $\alpha+2 \beta=n$. If $\alpha=0$, then $C$ are quaternary linear codes over $Z_{4}$ and if $\beta=0, C$ are equivalent to binary linear codes. In 2014, Abualrub et al. studied $Z_{2} Z_{4}$-additive cyclic codes and $Z_{2}+u Z_{2}$-linear cyclic codes [7]. In 2015, Aydogdu et al. studied the $Z_{2} Z_{2}[u]$-additive codes [8]. In 2016, Borges et al. researched the generator polynomials and dual codes of
$Z_{2} Z_{4}$-additive codes [9]. One year later, Aydogdu et al. studied the $Z_{2} Z_{4} Z_{8}$-cyclic codes [10], in which they introduced that if $\alpha, \beta$ and $\theta$ are odd integers, $Z_{2} Z_{4} Z_{8}$ cyclic codes is a $Z_{8}$-submodule of $Z_{2} /\left(x^{\alpha}-1\right) \times Z_{4} /\left(x^{\beta}-1\right) \times Z_{8} /\left(x^{\theta}-1\right)$ and give the minimal generating set for $Z_{2} Z_{4} Z_{8}$-cyclic code. In 2018, the binary images of $Z_{2} Z_{4}$-additive cyclic codes have been done also by Borges et al. [11]. Further, Ismail et al. discussed the structure properties of Z2Z2s-additive cyclic codes [12].

In this paper, we will study some structure of $Z_{2}\left(Z_{2}+u Z_{2}\right)\left(Z_{2}+u Z_{2}+u^{2} Z_{2}\right)$-additive cyclic codes, including their generator polynomials and spanning sets. The rest of this paper is organized as follows. In Section 2, some results on $Z_{2}\left(Z_{2}+u Z_{2}\right)\left(Z_{2}+u Z_{2}+u^{2} Z_{2}\right)$-additive code are given, and associate these codes with submodules of $Z_{2}{ }^{\alpha} \times\left(Z_{2}+u Z_{2}\right)^{\beta} \times\left(Z_{2}+u Z_{2}+u^{2} Z_{2}\right)^{\theta}$. Moreover, the generator matrices and parity-check matrices of $Z_{2}\left(Z_{2}+u Z_{2}\right)\left(Z_{2}+u Z_{2}+u^{2} Z_{2}\right)$-additive codes are given in this section. In Section 3 , the definition $Z_{2}\left(Z_{2}+u Z_{2}\right)\left(Z_{2}+u Z_{2}+u^{2} Z_{2}\right)$-additive cyclic codes and their generator polynomials and spanning sets are given. Some examples are given to illustrate the main results appeared in this paper. Moreover, using the Gray map, some good binary linear codes can be obtained by 
$Z_{2}\left(Z_{2}+u Z_{2}\right)\left(Z_{2}+u Z_{2}+u^{2} Z_{2}\right)$-additive cyclic codes.

\section{2. $Z_{2}\left(Z_{2}+u Z_{2}\right)\left(Z_{2}+u Z_{2}+u^{2} Z_{2}\right)$-Additive Codes}

Let $Z_{2}$ be the finite field of order 2, and let $R$ be the ring $Z_{2}+u Z_{2}=\{0,1, u, 1+u\}$, where $u^{2}=0 \bmod 2$, and let $S$ be the ring $Z_{2}+u Z_{2}+u^{2} Z_{2}=\left\{0,1, u, 1+u, u^{2}, 1+u^{2}, u+u^{2}, 1+u+u^{2}\right\}$, where $u^{2}=0 \bmod 2$. Construct the following set

$$
\begin{aligned}
& \Re=Z_{2}^{\alpha} \times\left(Z_{2}+u Z_{2}\right)^{\beta} \times\left(Z_{2}+u Z_{2}+u^{2} Z_{2}\right)^{\theta} \\
& =\left\{(m, v, w) \mid m \in Z_{2}^{\alpha}, v \in\left(Z_{2}+u Z_{2}\right)^{\beta}, w \in\left(Z_{2}+u Z_{2}+u^{2} Z_{2}\right)^{\theta}\right\}
\end{aligned}
$$

Clearly, this set is closed under usual addition and it becomes an additive abelian group. Define the following multiplication for $(m, v, w) \in \Re$ and $d \in S$, in order to make $\Re$ closed under multiplication by elements from $\mathrm{S}$,

$$
d \cdot(m, v, w)=\left(d m \bmod u, d v \bmod u^{2}, d w\right)
$$

Since $d \cdot(m, v, w) \in S$, it follows that $\Re$ is a $S$-module with respect to this scalar multiplication.

Definition 1 A non-empty subset $\mathrm{C}$ of $Z_{2}^{\alpha} \times R_{2}^{\beta} \times S_{2}^{\theta}$ is called a $Z_{2} R S$-additive codes if it is a subgroup of $Z_{2}^{\alpha} \times R_{2}^{\beta} \times S_{2}^{\theta}$.

Clearly, $C$ is a binary linear code of length $\alpha$ if $\beta=0$ and $\theta=0$, a $Z_{2} R$-additive code of length $(\alpha, \beta)$ if $\theta=0$ and a linear code of length $\theta$ over $S$ if $\alpha=0$ and $\beta=0$. The type of a $Z_{2} R S$-additive code of block length $(\alpha, \beta, \theta)$ is defined as follows.

$$
\Phi(m, v, w)=\left(m_{0}, \ldots, m_{\alpha-1}, \phi_{1}\left(v_{0}\right), \ldots, \phi_{1}\left(v_{\beta-1}\right), \phi_{2}\left(w_{0}\right), \ldots, \phi_{2}\left(w_{\theta-1}\right)\right)
$$

where

$$
m=\left(m_{0}, m_{1}, \ldots, m_{\alpha-1}\right) \in Z_{2}^{\alpha}, v=\left(v_{0}, v_{1}, \ldots, v_{\beta-1}\right) \in\left(Z_{2}+u Z_{2}\right)^{\beta}
$$$$
w=\left(w_{0}, w_{1}, \ldots, w_{\theta-1}\right) \in\left(Z_{2}+u Z_{2}+u^{2} Z_{2}\right)^{\theta}
$$

Definition 3 The Gray image $\Phi(C)=C$ of a $Z_{2} R S$ additive code $C$ is a binary code of length $n=\alpha+2 \beta+3 \theta$ and is called a $Z_{2} R S$-linear code.

Let $F_{q}$ is the finite field with $q$ elements, a linear code of length $n$ over $F_{q}$ is a linear subspace of the vector space $F_{q}{ }^{n}$.

The generator matrix of linear codes can be formed by the minimal spanning set of this linear code. Since the minimum spanning set of the linear codes is not unique, then the generator matrix of the linear codes is not unique. In this following, the paper gives the standard form of generator matrix of $Z_{2} R S$-additive codes first.

Theorem 1 Let $C$ be a $Z_{2} R S$-additive code of type $\left(\alpha, \beta, \theta ; k_{0} ; k_{1}, k_{2} ; k_{3}, k_{4}, k_{5}\right)$. Then $\mathrm{C}$ is permutation equivalent to a $Z_{2} R S$-additive code with the standard form matrix.
Definition $2 \mathrm{~A} Z_{2} R S$-additive code $C$ of length $(\alpha, \beta, \theta)$ is called $Z_{2} R S$-additive code of type $\left(\alpha, \beta, \theta ; k_{0} ; k_{1}, k_{2} ; k_{3}, k_{4}, k_{5}\right)$, if $C$ is a group isomorphic to the abelian structure

$$
Z_{2}^{k_{0}} \times R^{k_{1}} \times Z_{2}^{l_{2}} \times S^{k_{3}} \times R^{k_{4}} \times Z_{2}^{k_{5}}
$$

From [13, 14], defind $\phi_{1}: Z_{2}+u Z_{2} \rightarrow Z_{2}^{2} \quad$ by $\phi_{1}(0)=(0,0), \quad \phi_{1}(1)=(0,1), \quad \phi_{1}(u)=(1,1), \quad \phi_{1}(1+u)=(1,0)$ and $\phi_{2}: Z_{2}+u Z_{2}+u^{2} Z_{2} \rightarrow Z_{2}^{3} \quad$ by $\phi_{2}(0)=(0,0,0) \quad$, $\phi_{2}(1)=(0,1,1) \quad, \quad \phi_{2}(u)=(0,0,1) \quad, \quad \phi_{2}(1+u)=(0,1,0) \quad$ ， $\phi_{2}\left(u^{2}\right)=(1,1,0), \phi_{2}\left(1+u^{2}\right)=(1,0,1), \quad \phi_{2}\left(u+u^{2}\right)=(1,1,1)$, $\phi_{2}\left(1+u+u^{2)}=(1,0,0)\right.$.

By the maps $\phi_{1}$ and $\phi_{2}$, Gray map can be defined by $\Phi: Z_{2}^{\alpha} \times R^{\beta} \times S^{\theta} \rightarrow Z_{2}^{n}$ by: 


$$
u \cdot v=u^{2}\left(\sum_{i=1}^{\alpha} u_{i} v_{i}\right)+u\left(\sum_{j=\alpha+1}^{\alpha+\beta} u_{j} v_{j}\right)+\sum_{k=\alpha+\beta+1}^{\alpha+\beta+\theta} u_{k} v_{k}
$$

The dual code $C^{\perp}$ can be defined in usual way with respect to the inner product.

$$
C^{\perp}=\left\{u \in Z_{2}^{\alpha} \times R^{\beta} \times S^{\theta} \mid u \cdot v=0 \text { forallu } \in C\right\}
$$

It is obviously that $\mathrm{C}^{\perp}$ is also a $Z_{2} R S$-additive code.

Corollary 1 Let $C$ be a $Z_{2} R S$-additive code of type with standard form generator matrix (7). Then, the code $C^{\perp}$ is of type

$$
\left(\alpha, \beta, \theta ; \alpha-k_{0} ; \beta-k_{1}-k_{2}, k_{2} ; \theta-k_{3}-k_{4}-k_{5}, k_{5}, k_{4}\right)
$$

\section{3. $\mathrm{Z}_{2}$ RS-Additive Cyclic Codes}

Cyclic codes are a very significant class of linear codes because of their good algebraic structures for coding and decoding [15]. In this section, we study the structural properties of $Z_{2} R S$-additive cyclic codes, including their generator polynomials and minimal spanning sets.

Let $\delta$ be a standard shift operator on $Z_{2}^{\alpha}, R^{\beta}$ and $S^{\theta}$. For any $(m, v, w) \in Z_{2}^{\alpha} \times R^{\beta} \times S^{\theta}$, let $\tau$ be a shift operator on

$$
Z_{2}^{\alpha} \times R^{\beta} \times S^{\theta} \text { as } \tau(m, v, w)=(\delta(m), \delta(v), \delta(w))
$$

Definition 4 The $Z_{2} R S$-additive code $\mathrm{C}$ of length $n=\alpha+\beta+\theta$ over $\Re$ is said to be a $Z_{2} R S$-additive cyclic code if it is invariant under $\tau$, i.e. for any codeword,

$$
c=\left(m_{0}, m_{1}, \cdots, m_{\alpha-1}, v_{0}, v_{1}, \cdots, v_{\beta-1}, w_{0}, w_{1}, \cdots, w_{\theta-1}\right) \in C
$$

its cyclic shift

$$
\tau(c)=\left(m_{\alpha-1}, m_{0}, \cdots, m_{\alpha-2}, v_{\beta-1}, v_{0}, \cdots, v_{\beta-2}, w_{\theta-1}, w_{0}, \cdots w_{\theta-2}\right) \in C
$$

In Section 2, the paper defined the inner product in $Z_{2}^{\alpha} \times R^{\beta} \times S^{\theta}$

Lemma 1 If $C$ is a $Z_{2} R S$-additive cyclic code, then $C^{\perp}$ is also a $Z_{2} R S$-additive cyclic code.

Proof Let $\mathrm{C}$ be a $Z_{2} R S$-additive cyclic code, and

$$
m=\left(a_{0}, \cdots a_{\alpha-} 1, b_{0}, \cdots, b_{\beta-1}, d_{0}, \cdots d_{\theta-1}\right) \in C^{\perp}
$$

In the following, it only needs to prove $\tau(m) \in C^{\perp}$. Since $m \in C^{\perp}$, for

$$
v=\left(e_{0}, \cdots, e_{\alpha-1}, g_{0}, \cdots, g_{\beta-1}, h_{0}, h_{\theta-1}\right) \in C
$$

it have

$$
\begin{aligned}
& m \cdot v=u^{2}\left(a_{0} e_{0}+\cdots+a_{\alpha-1} e_{\alpha-1}\right)+u\left(b_{0} g_{0}+\cdots+b_{\beta-1} g_{\beta-1}\right) \\
& +d_{0} h_{0}+\cdots+d_{\theta-1} h_{\theta-1} \equiv 0 \bmod u^{3}
\end{aligned}
$$

Let

$$
l=l c m(\alpha, \beta, \theta)
$$

and

$$
\tau^{l-1}(v)=\left(e_{1}, \ldots, e_{\alpha-1}, e_{0}, g_{1}, \ldots, g_{\beta-1}, g_{0}, h_{1}, \ldots, h_{\theta-1}, h_{0}\right)=w
$$

Then $\tau^{l}=v$. Since $C$ is a cyclic code, it follows that $w \in C$. Therefore,

$$
\begin{aligned}
& 0=w \cdot m \\
& =u^{2}\left(e_{1} a_{0}+\cdots+e_{\alpha-1} a_{\alpha-2}+e_{0} a_{\alpha-1}\right)+u\left(g_{1} b_{0}+\cdots+g_{0} b_{\beta-1}\right) \\
& +h_{1} d_{0}+h_{0} d_{\theta-1} \\
& =u^{2}\left(e_{0} a_{\alpha-1}+\cdots+e_{\alpha-1} a_{\alpha-2}\right)+u\left(g_{0} b \beta-1++g_{\beta-1} b_{\beta-2}\right) \\
& +h_{0} d_{\theta-1}++h_{\theta-1} d_{\theta-2} \\
& =v \cdot \tau(m) .
\end{aligned}
$$

Hence, $\tau(u) \in C, C^{\perp}$ is also a $Z_{2} R S$-additive cyclic code.

Definition 5 Define the set

$$
Z_{2}[x] /\left(x^{\alpha}-1\right) \times R[x] /\left(x^{\beta}-1\right) \times S[x] /\left(x^{\theta}-1\right) \text { by } \Re_{\alpha, \beta, \theta} \text {. }
$$

For $C \subseteq Z_{2}^{\alpha} \times R^{\beta} \times S^{\theta}$ any elenment:

$$
c=\left(m_{0}, m_{1}, \cdots, m_{\alpha-1}, v_{0}, v_{1}, \cdots, v_{\beta-1}, w_{0}, w_{1}, \cdots, w_{\theta-1}\right) \in C,
$$

can be written by the element of $\Re_{\alpha, \beta, \theta}$ as follows

$$
\begin{aligned}
& c(x)=\left(m_{0}+m_{1} x+\cdots+m_{\alpha-1} x^{\alpha-1}, v_{0}+v_{1} x+\cdots+v_{\beta-1} x^{\beta-1},\right. \\
& \left.w_{0}+w_{1}+\cdots+w_{\theta-1} x^{\theta-1}\right)=(m(x), v(x), w(x)) .
\end{aligned}
$$

Therefore, it is possible to see that $\Re_{\alpha, \beta, \theta} \cong Z_{2}^{\alpha} \times R^{\beta} \times S^{\theta}$. Define the product $*$, for $d(x) \in S[x]$ and $(f(x), g(x), h(x)) \in \Re_{\alpha, \beta, \theta}$,

$$
\begin{aligned}
& d(x) *(f(x,) g(x), h(x)) \\
& =\left(d(x) f(x) \bmod u, d(x) g(x) \bmod u^{2}, d(x) h(x)\right)
\end{aligned}
$$

This extended multiplication is also well defined and $Z_{2}^{\alpha} \times R^{\beta} \times S^{\theta}$ is a $S[x]$-module with respect to this multiplication.

Lemma 2 Under the definition of $*, \Re_{\alpha, \beta, \theta}$ is an $S[x]$ module, and any $Z_{2} R S$-additive cyclic code $C$ corresponds to an $S[x]$-submodule of $\Re_{\alpha, \beta, \theta}$.

In this part, we study $S[x]$-submodules for $\mathfrak{R}_{\alpha, \beta, \theta}$. The generator and a minimal spanning set of these submodules are determined. Assume that $\alpha, \beta$, and $\theta$ are odd positive integers. Since the $Z_{2} R S$-additive cyclic code $C$ is an $S[x]$ 
-submodule of $\Re_{\alpha, \beta, \theta}$, a map can be defined as follows

$$
\begin{aligned}
& \psi: C \rightarrow S[x] /\left(x^{\theta}-1\right) \\
& (f(x), g(x), h(x)) \rightarrow h(x)
\end{aligned}
$$

Clearly, $\psi$ is an $S[x]$-module homomorphism with kernel

$$
\begin{aligned}
& \operatorname{Ker}(\psi)=\left\{(f(x), g(x), 0) \in C: f(x) \in Z_{2}[x] /\left(x^{\alpha}-1\right),\right. \\
& \left.g(x) \in R[x] /\left(x^{\beta}-1\right)\right\}
\end{aligned}
$$

Since the image $\psi(C)$ of $\mathrm{C}$ is an ideal of $S[x] /\left(x^{\theta}-1\right)$. and $\theta$ is an odd integer, which implies that

$$
\psi(C)=\left\langle p(x)+u q(x)+u^{2} r(x)\right\rangle
$$

where $r(x)|q(x)| p(x) \mid\left(x^{\theta}-1\right) \bmod u^{3}$.

From the discussion above, which implies that the following theorem is got.

Theorem 3 Let $C$ be a $Z_{2} R S$-additive cyclic code. Then $C$ can be generated as an $R[x]$-submodule of $\Re_{\alpha, \beta, \theta}$ with this form

$$
\begin{aligned}
& C=\left\langle(f(x), 0,0),\left(f_{1}(x), g_{1}(x)+u a_{1}(x), 0\right),\right. \\
& \left.\left(f_{2}(x), g_{2}(x), p(x)+u q(x)+u^{2} r(x)\right)\right\rangle .
\end{aligned}
$$

Proof Let $I$ be a set, and

$$
\begin{aligned}
& I=\left\{(f(x), g(x), 0) \in Z_{2}[x] /\left(x^{\alpha}-1\right) \times R[x] /\left(x^{\beta}-1\right):\right. \\
& (f(x), g(x), 0) \in \operatorname{ker}(\psi)\}
\end{aligned}
$$

Clearly, $\quad I$ is an $R[x]$-submodule of $Z_{2}[x] /\left\langle x^{\alpha}-1\right\rangle \times R[x] /\left\langle x^{\beta}-1\right\rangle$. Hence, $I$ is a $Z_{2} R S$ additive cyclic code. From [7],

$$
I=\left\langle(f(x), 0),\left(f_{1}(x), g_{1}(x)+u a_{1}(x)\right\rangle,\right.
$$

where $f(x) \mid\left\langle x^{\alpha}-1\right\rangle$ and $g_{1}(x)+u a_{1}(x)$ is a polynomial over $R$ with $a_{1}(x)\left|g_{1}(x)\right|\left\langle x^{\beta}-1\right\rangle$ and $f(x) \mid \frac{x^{\beta}-1}{a_{1}(x)} f_{1}(x)$.

Let $\left(c_{1}(x), c_{2}(x), 0\right) \in \operatorname{ker}(\psi)$. Then that have

$$
\begin{aligned}
& \left(c_{1}(x), c_{2}(x)\right) \in I \\
& =\left\langle(f(x), 0),\left(f_{1}(x), g_{1}(x)+u a_{1}(x)\right)\right\rangle
\end{aligned}
$$

Therefore, for polynomials $m_{1} \in Z_{2}[x] /\left\langle x^{\alpha}-1\right\rangle$ and $m_{2} \in R[x] /\left\langle x^{\beta}-1\right\rangle$, it get

$$
\left(c_{1}(x), c_{2}(x)\right)=m_{1} *(f(x), 0)+m_{2} *\left(f_{1}(x), g_{1}(x)+u a_{1}(x)\right)
$$

So

$$
\operatorname{ker}(\psi)=\left\langle(f(x), 0,0),\left(f_{1}(x), g_{1}(x)+u a_{1}(x), 0\right\rangle\right.
$$

is a submodule of $C$. By the first isomorphism theorem of modules, there are $C / \operatorname{ker}(\psi) \cong\left\langle p(x)+u q(x)+u^{2} r(x)\right\rangle$.

Let $\left(f_{2}(x), g_{2}(x), p(x)+u q(x)+u^{2} r(x)\right) \in C$ such that

$$
\begin{aligned}
& \psi\left(f_{2}(x), g_{2}(x), p(x)+u q(x)+u^{2} r(x)\right) \\
& =p(x)+u q(x)+u^{2} r(x)
\end{aligned}
$$

Then

$$
\begin{aligned}
& C=\left\langle(f(x), 0,0),\left(f_{1}(x), g_{1}(x)+u a_{1}(x), 0\right),\right. \\
& \left.\left(f_{2}(x), g_{2}(x), p(x)+u q(x)+u^{2} r(x)\right)\right\rangle .
\end{aligned}
$$

Lemma 3 Let

$$
\begin{aligned}
& C=\left\langle(f(x), 0,0),\left(f_{1}(x), g_{1}(x)+u a_{1}(x), 0\right),\right. \\
& \left.\left(f_{2}(x), g_{2}(x), p(x)+u q(x)+u^{2} r(x)\right)\right\rangle
\end{aligned}
$$

be a $Z_{2} R S$-additive cyclic code. Then

$$
\operatorname{deg} f_{1}(x)<\operatorname{deg} f(x)
$$

and $\operatorname{deg} f_{2}(x)<\operatorname{deg} f(x)$ and

$$
\operatorname{deg} g_{2}(x)<\operatorname{deg} g_{1}(x)
$$

Proof Let $\operatorname{deg} f_{1}(x) \geq \operatorname{deg} f(x)$. Since $f(x)$ is monic, it can apply division algorithm, i.e. there exist polynomials $q^{\prime}(x)$ and $r^{\prime}(x)$ over $Z_{2}$ such that

$$
f_{1}(x)=f(x) q^{\prime}(x)+r^{\prime}(x)
$$

where $r^{\prime}(x)=0$ or $0 \leq \operatorname{deg} r^{\prime}(x)<\operatorname{deg} f(x)$, which implies that

$$
\begin{aligned}
& \left\langle(f(x), 0,0),\left(f_{1}(x), g_{1}(x)+u a_{1}(x), 0\right),\right. \\
& \left.\left(f_{2}(x), g_{2}(x), p(x)+u q(x)+u^{2} r(x)\right)\right\rangle \\
& =\left\langle(f(x), 0,0),\left(f(x) q^{\prime}(x)+r^{\prime}(x), g_{1}(x)+u a_{1}(x), 0\right),\right. \\
& \left.\left(f_{2}(x), g_{2}(x), p(x)+u q(x)+u^{2} r(x)\right)\right\rangle \\
& =\left\langle(f(x), 0,0),\left(r^{\prime}(x), g_{1}(x)+u a_{1}(x), 0\right),\right. \\
& \left.\left(f_{2}(x), g_{2}(x), p(x)+u q(x)+u^{2} r(x)\right)\right\rangle .
\end{aligned}
$$

Hence we may assume that $\operatorname{deg} f_{1}(x)<\operatorname{deg} f(x)$. Similarly the $\operatorname{deg} f_{2}(x)<\operatorname{deg} f(x) \quad$ and $\operatorname{deg} g_{2}(x)<\operatorname{deg} g_{1}(x)$ also can be proved.

Lemma 4 Let

$$
\begin{aligned}
& C=\left\langle(f(x), 0,0),\left(f_{1}(x), g_{1}(x)+u a_{1}(x), 0\right),\right. \\
& \left.\left(f_{2}(x), g_{2}(x), p(x)+u q(x)+u^{2} r(x)\right)\right\rangle .
\end{aligned}
$$

be a $Z_{2} R S$-additive cyclic code. Then 


$$
f(x) \mid \frac{x^{\beta}-1}{a_{1}(x)} f_{1}(x)
$$

Proof It is well know that

$$
\psi(C)=\left\langle p(x)+u q(x)+u^{2} r(x)\right\rangle
$$

Hence,

$$
\begin{aligned}
& \psi\left(\frac{x^{\beta}-1}{a_{1}(x)} *\left(f_{1}(x), g_{1}(x)+u a_{1}(x), 0\right)\right) \\
& \left.=\psi\left(\delta\left(\frac{x^{\beta}-1}{a_{1}(x)}\right) f_{1}(x), \delta\left(\frac{x^{\beta}-1}{a_{1}(x)}\right)\left(g_{1}(x)+u a_{1}(x)\right), 0\right)\right) \\
& =0
\end{aligned}
$$

where $\left(f_{1}(x), g_{1}(x)+u a_{1}(x), 0\right) \in C$, which implies that

$$
\delta\left(\frac{x^{\beta}-1}{a_{1}(x)}\right) f_{1}(x)=\left(\frac{x^{\beta}-1}{a_{1}(x)}\right) f_{1}(x) \in \operatorname{ker}(\psi)
$$

Therefore,

$$
f(x) \mid \frac{x^{\beta}-1}{a_{1}(x)} f_{1}(x)
$$

\section{Lemma 5 Let}

$$
\begin{aligned}
& C=\left\langle(f(x), 0,0),\left(f_{1}(x), g_{1}(x)+u a_{1}(x), 0\right),\right. \\
& \left.\left(f_{2}(x), g_{2}(x), p(x)+u q(x)+u^{2} r(x)\right)\right\rangle .
\end{aligned}
$$

be a $Z_{2} R S$-additive cyclic code. Then have

$$
\begin{aligned}
& \left(g_{1}(x)+u a_{1}(x)\right) \mid \frac{x^{\theta}-1}{r(x)} g_{2}(x) \bmod u^{2} \\
& f(x) \mid\left(k(x) f_{1}(x)+\frac{x^{\theta}-1}{r(x)} f_{2}(x)\right) \bmod u
\end{aligned}
$$

Where

$$
k(x)\left(g_{1}(x)+u a_{1}(x)\right)=\frac{x^{\theta}-1}{r(x)} g_{2}(x) \bmod u^{2}
$$

\section{Proof (i) Consider}

$$
\begin{aligned}
& \psi\left(\frac{x^{\theta}-1}{r(x)} *\left(f_{2}(x), g_{2}(x), p(x)+u q(x)+u^{2} r(x)\right)\right) \\
& \left.=\psi\left(\delta\left(\frac{x^{\theta}-1}{r(x)}\right) f_{2}(x), \delta\left(\frac{x^{\theta}-1}{r(x)}\right) g_{2}(x), 0\right)\right) \\
& =0 .
\end{aligned}
$$

$$
\delta\left(\frac{x^{\theta}-1}{r(x)}\right) g_{2}(x)=\frac{x^{\theta}-1}{r(x)} g_{2}(x) \in \operatorname{ker}(\psi)
$$

then

$$
\left(g_{1}(x)+u a_{1}(x)\right) \mid \frac{x^{\theta}-1}{r(x)} g_{2}(x) \bmod u^{2}
$$

(ii) Let

$$
\left(g_{1}(x)+u a_{1}(x)\right) \mid \frac{x^{\theta}-1}{r(x)} g_{2}(x) \bmod u^{2}
$$

Then there exists $k(x) \in S$ such that

$$
k(x)\left(g_{1}(x)+u a_{1}(x)\right)=\frac{x^{\theta}-1}{r(x)} g_{2}(x) \bmod u^{2}
$$

Having

$$
\begin{aligned}
& k(x) *\left(f_{1}(x), g_{1}(x)+u a_{1}(x), 0\right) \\
& =\left(k(x) f_{1}(x), k(x)\left(g_{1}(x)+u a_{1}(x), 0\right)\right) \in C
\end{aligned}
$$

Therefore,

$$
\begin{aligned}
& \left(k(x) f_{1}(x)+k(x)\left(g_{1}(x)+u a_{1}(x)\right), 0\right) \\
& -\left(\frac{x^{\theta}-1}{r(x)} f_{2}(x), \frac{x^{\theta}-1}{r(x)} g_{2}(x), 0\right) \\
& =\left(k(x) f_{1}(x)+\frac{x^{\theta}-1}{r(x)} f_{2}(x), 0,0\right) \in \operatorname{ker}(\psi) \subseteq C .
\end{aligned}
$$

Hence,

$$
f(x) \mid\left(k(x) f_{1}(x)+\frac{x^{\theta}-1}{r(x)} f_{2}(x)\right) \bmod u
$$

From the discussion above, the following theorem can be directly obtained.

Theorem 4 Let $C$ be a $Z_{2} R S$-additive cyclic code. Then,

$$
C=\langle(f(x), 0,0)\rangle, f(x) \mid\left(x^{\alpha}-1\right) \bmod u
$$

or

$$
C=\left\langle f_{1}(x), g_{1}(x)+u a_{1}(x), 0\right\rangle, a_{1}(x)\left|g_{1}(x)\right|\left(x^{\beta}-1\right) \bmod u^{2}
$$
and

$$
\left(x^{\alpha}-1\right) \mid\left(\frac{x^{\beta}-1}{a_{1}(x)}\right) f_{1}(x)
$$

or

$$
\begin{aligned}
& C=\left\langle(f(x), 0,0),\left(f_{1}(x), g_{1}(x)+u a_{1}(x), 0\right),\right. \\
& \left.\left(f_{2}(x), g_{2}(x), p(x)+u q(x)+u^{2} r(x)\right)\right\rangle,
\end{aligned}
$$

where 


$$
\begin{gathered}
r(x)|q(x)| p(x) \mid\left(x^{\theta}-1\right) \bmod u^{3} \\
\operatorname{deg} g_{2}(x)<\operatorname{deg} g_{1}(x)
\end{gathered}
$$

and

$$
\left(g_{1}(x)+u a_{1}(x)\right) \mid \frac{x^{\theta}-1}{a_{1}(x)} g_{2}(x) \bmod u^{2}
$$

$\operatorname{deg} f_{1}(x)<\operatorname{deg} f(x), \operatorname{deg} f_{2}(x)<\operatorname{deg} f(x)$

and

$$
\begin{gathered}
f(x) \mid\left(\frac{x^{\beta}-1}{a_{1}(x)}\right) f_{1}(x) \bmod u \\
f(x) \mid\left(k(x) f_{1}(x)+\frac{x-1}{r(x)} f_{2}(x)\right) \bmod u
\end{gathered}
$$

where

$$
k(x)\left(g_{1}(x)+a_{1}(x)\right)=\frac{x^{\theta}-1}{r(x)} g_{2}(x) \bmod u^{2}
$$

In the following, get the main result on the minimum generating sets of $Z_{2} R S$-additive cyclic codes.

Theorem 5 Let

$$
\begin{aligned}
& C=\left\langle(f(x), 0,0),\left(f_{1}(x), g_{1}(x)+u a_{1}(x), 0\right),\right. \\
& \left.\left(f_{2}(x), g_{2}(x), p(x)+u q(x)+u^{2} r(x)\right)\right\rangle .
\end{aligned}
$$

Let,

$$
\begin{aligned}
& D_{1}=\bigcup_{i=0}^{\alpha-t_{1}-1}\left\{x^{i} *(f(x), 0,0)\right\}, \\
& D_{2}=\bigcup_{i=0}^{\beta-t_{2}-1}\left\{x^{i} *\left(f_{1}(x), g_{1}(x)+u a_{1}(x), 0\right)\right\}, \\
& D_{3}=\bigcup_{i=0}^{\theta-t_{3}-1}\left\{x^{i} *\left(f_{2}(x), g_{2}(x), p(x)+u q(x)+u^{2} r(x)\right)\right\}, \\
& D_{4}=\bigcup_{i=0}^{\operatorname{deg}\left(a_{1}(x)\right)-1}\left\{x^{i} *\left(l_{g_{1}}(x) f_{1}(x), u l_{g 1}(x) a_{1}(x), 0\right)\right\}, \\
& D_{5}=\bigcup_{i=0}^{\operatorname{deg}(q(x))-1}\left\{x^{i} *\left(l_{p}(x) f_{2}(x), l_{p}(x) g_{2}(x), l_{p}\left(u q(x)+u^{2} r(x)\right)\right)\right\}, \\
& D_{6}=\bigcup_{i=0}^{\operatorname{deg}\left(r^{r}(x)\right)-1}\left\{x^{i} *\left(l_{p}(x) f_{2}(x), l_{p}(x) g_{2}(x), u^{2} l_{q}(x) r(x)\right)\right\},
\end{aligned}
$$

where $\quad \operatorname{deg} f(x)=t_{1}, \quad, \quad \operatorname{deg} g_{1}(x)=t_{2}, \quad \operatorname{deg} p(x)=t_{3}$, $a_{1}^{\prime}(x)=\frac{g_{1}(x)}{a_{1}(x)}, \quad q^{\prime}(x)=\frac{p(x)}{q(x)}, r^{\prime}(x)=\frac{q(x)}{r(x)}, l_{f}(x) f(x)=x^{\alpha}-1$, $l_{g 1}(x) g_{1}(x)=x^{\beta}-1 \quad, \quad l_{p}(x) p(x)=l_{q}(x) q(x)=x^{\theta}-1$. Then

$$
D=D_{1} \cup D_{2} \cup D_{3} \cup D_{4} \cup D_{5} \cup D_{6}
$$

is a minimal spanning set for $C$, and

$$
|C|=2^{\alpha-t_{1}} \cdot 4^{\beta-t_{2}} \cdot 8^{\theta-t_{3}} \cdot 2^{\operatorname{deg} a_{1}^{\prime}(x)} \cdot 4^{\operatorname{deg} q^{\prime}(x)} \cdot 2^{\operatorname{deg} r^{\prime}(x)}
$$

Proof Let $c(x)$ be a any codeword in $C$. There exist some polynomials $e_{1}(x), e_{2}(x), e_{3}(x) \in S[x]$, such that $c(x)$ can be represented by

$$
\begin{aligned}
& c(x)=e_{1}(x) *(f(x), 0,0)+e_{2}(x) *\left(f_{1}(x), g_{1}(x)+u a_{1}(x), 0\right) \\
& +e_{3}(x) *\left(f_{2}(x), g_{2}(x), p(x)+u q(x)+u^{2} r(x)\right)
\end{aligned}
$$

If

$\operatorname{deg} e_{1}(x)<\operatorname{deg} l_{f}(x)$, then

$$
e_{1}(x) *(f(x), 0,0) \in\left\langle D_{1}\right\rangle
$$

Otherwise, have

$$
e_{1}(x)=l_{f}(x) q_{1}(x)+r_{1}(x)
$$

where $r_{1}(x)=0$ or $\operatorname{deg} r_{1}(x)<\operatorname{deg} l_{f}(x)$. Hence,

$$
\begin{aligned}
& e_{1}(x) *(f(x), 0,0)=l_{f}(x) q_{1}(x)+r_{1}(x) *(f(x), 0,0) \\
& =r_{1}(x) *(f(x), 0,0) \in\left\langle D_{1}\right\rangle .
\end{aligned}
$$

If $\operatorname{deg} e_{2}(x)<\operatorname{deg} l_{f}(x)$, then

$$
e_{2}(x) *\left(f_{1}(x), g_{1}(x)+u a_{1}(x), 0\right) \in\left\langle D_{2}\right\rangle
$$

Otherwise, have

$$
e_{2}(x)=l_{g 1}(x) q_{2}(x)+r_{2}(x)
$$

where $r_{2}(x)=0$ or $\operatorname{deg} r_{2}(x)=\operatorname{deg} l_{g_{1}}(x)$. Hence,

$$
\begin{aligned}
& e_{2}(x) *\left(f_{1}(x), g_{1}(x)+u a_{1}(x), 0\right) \\
& =l_{g 1}(x) q_{2}(x)+r_{2}(x) *\left(f_{1}(x), g_{1}(x)+u a_{1}(x), 0\right) \\
& =l_{g 1}(x) q_{2}(x) *\left(f_{1}(x), g_{1}(x)+u a_{1}(x), 0\right) \\
& +r_{2}(x) *\left(f_{1}(x), g_{1}(x)+u a_{1}(x), 0\right) \\
& =q_{2}(x) *\left(l_{g 1}(x) f_{1}(x), l_{g 1}(x) u a_{1}(x), 0\right) \\
& +r_{2}(x) *\left(f_{1}(x), g_{1}(x)+u a_{1}(x), 0\right)
\end{aligned}
$$

where $r_{2}(x) *\left(f_{1}(x), g_{1}(x)+u a_{1}(x), 0\right) \in\left\langle D_{2}\right\rangle$.

For

$$
q_{2}(x) *\left(l_{g 1}(x) f_{1}(x), l_{g 1}(x) u a_{1}(x), 0\right)
$$

if $\operatorname{deg} q_{2}(x)<\operatorname{deg} a_{1}^{\prime}(x)$, then

$$
q_{2}(x) *\left(l_{g 1}(x) f_{1}(x), u l_{g 1}(x) a_{1}(x), 0\right) \in\left\langle D_{4}\right\rangle
$$


Otherwise, have

$$
q_{2}(x)=q_{3}(x) a_{1}^{\prime}(x)+r_{3}(x)
$$

where $r_{3}(x)=0$ or $\operatorname{deg} r_{3}(x)<\operatorname{deg} a_{1}^{\prime}(x)$. Hence,

$$
\begin{aligned}
& q_{2}(x) *\left(l_{g 1}(x) f_{1}(x), u l_{g 1}(x) a_{1}(x), 0\right) \\
& =\left(q_{3}(x) a_{1}^{\prime}(x)+r_{3}(x)\right) *\left(l_{g 1}(x) f_{1}(x), u l_{g 1}(x) a_{1}(x), 0\right) \\
& =q_{3}(x) *\left(l_{g 1}(x) f_{1}(x) a_{1}^{\prime}(x), 0,0\right) \\
& +r_{3}(x) *\left(l_{g 1}(x) f_{1}(x), u l_{g 1}(x) a_{1}(x), 0\right)
\end{aligned}
$$

Here,

$$
r_{3}(x) *\left(l_{g 1}(x) f_{1}(x), u l_{g 1}(x) a_{1}(x), 0\right) \in\left\langle D_{4}\right\rangle
$$

and since

$$
f(x) \mid \frac{x^{\beta}-1}{a_{1}(x)} f_{1}(x)
$$

$$
q_{3}(x) *\left(l_{g 1}(x) f_{1}(x) a_{1}^{\prime}(x), 0,0\right)=q_{3}(x) *\left(\frac{x^{\beta}-1}{a_{1}(x)} f_{1}(x), 0,0\right) \in\left\langle D_{1}\right\rangle
$$

Finally, for $e_{3}(x) *\left(f_{2}(x), g_{2}(x), p(x)+u q(x)+u^{2} r(x)\right)$, if $\operatorname{deg} e_{3}(x)<\operatorname{deg} l_{p}(x)$, then

$$
e_{3}(x)=l_{\mathrm{p}}(x) q_{4}(x)+r_{4}(x)
$$

$e_{3}(x) *\left(f_{2}(x), g_{2}(x), p(x)+u q(x)+u^{2} r(x)\right) \in\left\langle D_{1}\right\rangle$

where $r_{4}(x)=0$ or $\operatorname{deg} r_{4}(x)<\operatorname{deg} l_{p}(x)$.

Hence,

Otherwise,

$$
\begin{aligned}
& e_{3}(x) *\left(f_{2}(x), g_{2}(x), p(x)+u q(x)+u^{2} r(x)\right) \\
& =l_{\mathrm{p}}(x) q_{4}(x)+r_{4}(x) *\left(f_{2}(x), g_{2}(x), p(x)+u q(x)+u^{2} r(x)\right) \\
& =q_{4}(x) *\left(l_{\mathrm{p}}(x) f_{2}(x), l_{\mathrm{p}}(x) g_{2}(x), u l_{\mathrm{p}}(x) q(x)+u^{2} l_{\mathrm{p}}(x) r(x)\right) \\
& +r_{4}(x) *\left(f_{2}(x), g_{2}(x), p(x)+u q(x)+u^{2} r(x)\right) .
\end{aligned}
$$

Here,

$$
r_{4}(x) *\left(f_{2}(x), g_{2}(x), p(x)+u q(x)+u^{2} r(x)\right) \in\left\langle D_{3}\right\rangle
$$

For

$$
q_{4}(x) *\left(l_{\mathrm{p}}(x) f_{2}(x), l_{\mathrm{p}}(x) g_{2}(x), u l_{\mathrm{p}}(x) q(x)+u^{2} l_{\mathrm{p}}(x) r(x)\right)
$$

if $\operatorname{deg} q_{4}(x)<\operatorname{deg} q^{\prime}(x)$, then

$$
q_{4}(x) *\left(l_{\mathrm{p}}(x) f_{2}(x), l_{\mathrm{p}}(x) g_{2}(x), u l_{\mathrm{p}}(x) q(x)+u^{2} l_{\mathrm{p}}(x) r(x)\right) \in\left\langle D_{5}\right\rangle
$$

Otherwise, have

$$
q_{4}(x)=q^{\prime}(x) q_{5}(x)+r_{5}(x)
$$

where $r_{5}(x)=0$ or $\operatorname{deg} r_{5}(x)<\operatorname{deg} q^{\prime}(x)$.

Hence, 


$$
\begin{aligned}
& q_{4}(x) *\left(l_{\mathrm{p}}(x) f_{2}(x), l_{\mathrm{p}}(x) g_{2}(x), u l_{\mathrm{p}}(x) q(x)+u^{2} l_{\mathrm{p}}(x) r(x)\right) \\
& =q^{\prime}(x) q_{5}(x) \\
& +r_{5}(x) *\left(l_{\mathrm{p}}(x) f_{2}(x), l_{\mathrm{p}}(x) g_{2}(x), u l_{\mathrm{p}}(x) q(x)+u^{2} l_{\mathrm{p}}(x) r(x)\right) \\
& =q_{5}(x) *\left(l_{\mathrm{p}}(x) f_{2}(x), l_{\mathrm{p}}(x) g_{2}(x), u^{2} l_{\mathrm{p}}(x) r(x)\right) \\
& +r_{5}(x) *\left(l_{\mathrm{p}}(x) f_{2}(x), l_{\mathrm{p}}(x) g_{2}(x), u l_{\mathrm{p}}(x) q(x)+u^{2} l_{\mathrm{p}}(x) r(x)\right)
\end{aligned}
$$

Here,

In this following, consider the

$r_{5}(x) *\left(l_{\mathrm{p}}(x) f_{2}(x), l_{\mathrm{p}}(x) g_{2}(x), u l_{\mathrm{p}}(x) q(x)+u^{2} l_{\mathrm{p}}(x) r(x)\right) \in\left\langle D_{5}\right\rangle$.

For $q_{5}(x) *\left(l_{\mathrm{p}}(x) f_{2}(x), l_{\mathrm{p}}(x) g_{2}(x), u^{2} l_{\mathrm{p}}(x) r(x)\right), \quad$ if

$$
q_{6}(x) *\left(\frac{x^{\theta}-1}{r(x)} f_{2}(x), \frac{x^{\theta}-1}{r(x)} g_{2}(x), 0\right)
$$

$\operatorname{deg}\left(q_{5}(x)\right)<\operatorname{deg}\left(r^{\prime}(x)\right)$, then

$q_{5}(x) *\left(l_{\mathrm{p}}(x) f_{2}(x), l_{\mathrm{p}}(x) g_{2}(x), u^{2} l_{\mathrm{p}}(x) r(x)\right) \in\left\langle D_{6}\right\rangle$

Otherwise

$$
q_{5}(x)=r^{\prime}(x) q_{6}(x)+r_{6}(x)
$$

where $r_{6}(x)=0$ or $\operatorname{deg} r_{6}(x)<\operatorname{deg} r^{\prime}(x)$.

Hence,

$$
\begin{aligned}
& r^{\prime}(x) q_{6}(x)+r_{6}(x) *\left(l_{p}(x) f_{2}(x), l_{p}(x) g_{2}(x), u^{2} l_{p}(x) r(x)\right) \\
& =q_{6}(x) *\left(\frac{x^{\theta}-1}{r(x)} f_{2}(x), \frac{x^{\theta}-1}{r(x)} g_{2}(x), 0\right) \\
& +r_{6}(x) *\left(l_{p}(x) f_{2}(x), l_{p}(x) g_{2}(x), u^{2} l_{p}(x) r(x)\right)
\end{aligned}
$$

Have

$$
\begin{aligned}
r_{6}(x) *\left(l_{p}(x) f_{2}(x), l_{p}(x) g_{2}(x),\right. & \left.u^{2} l_{p}(x) r(x)\right) \in\left\langle D_{6}\right\rangle(96) . \quad \text { Hence, } \\
& q_{6}(x) *\left(\frac{x^{\theta}-1}{r(x)} f_{2}(x), \frac{x^{\theta}-1}{r(x)} g_{2}(x), 0\right) \\
& =q_{6}(x) *\left(f(x) \lambda(x)-k(x) f_{1}(x), k(x)\left(g_{1}(x)+u a_{1}(x), 0\right)\right) \\
& =q_{6}(x) \lambda(x)(f(x), 0,0)+q_{6}(x) k(x)\left(f_{1}(x), g_{1}(x)+u a_{1}(x), 0\right) \\
& \in\left\langle D_{1} \cup D_{2}\right\rangle .
\end{aligned}
$$

From lemma 5, there exists $k(x) \in S[x]$ such that

$$
k(x)\left(g_{1}(x)+u a_{1}(x)\right)=\frac{x^{\theta}-1}{r(x)} g_{2}(x) \bmod u^{2}
$$

and

$$
f(x) \mid\left(k(x) f_{1}(x)+\frac{x^{\theta}-1}{r(x)} f_{2}(x)\right) \bmod u
$$

Therefore, there is $\lambda(x)$ such that

$$
f(x) \lambda(x)=k(x) f_{1}(x)+\frac{x^{\theta}-1}{r(x)} f_{2}(x)
$$

which implies that,

$$
\frac{x^{\theta}-1}{r(x)} f_{2}(x)=f(x) \lambda(x)-k(x) f_{1}(x)
$$

Example 1 Let $C$ be a $Z_{2} R S$-additive cyclic code in generated $Z_{2}[x] /(x-1) \times R[x] /(x-1) \times S[x] /\left(x^{5}-1\right)$ by

$$
\begin{aligned}
& \left\langle(f(x), 0,0),\left(f_{1}(x), g_{1}(x)+u a_{1}(x), 0\right),\right. \\
& \left.\left(f_{2}(x), g_{2}(x), p(x)+u q(x)+u^{2} r(x)\right)\right\rangle,
\end{aligned}
$$

where $f(x)=1+x, f_{1}(x)=f_{2}(x)=1, f_{1}(x)=f_{2}(x)=1$, $g_{2}(x)=1, a_{1}(x)=1, p(x)=1+x, q(x)=1, r(x)=1$. In this following,

$$
\begin{aligned}
& f(x) l_{f}(x)=x-1 \Rightarrow l_{f}(x)=1, \\
& p(x) l_{p}(x)=x^{5}-1 \Rightarrow l_{p}(x)=x^{4}+x^{3}+x^{2}+1, \\
& q(x) l_{q}(x)=x^{5}-1 \Rightarrow l_{q}(x)=x^{5}-1, \\
& g_{1}(x) l_{g_{1}}(x)=x-1 \Rightarrow l_{g_{1}}(x)=1 .
\end{aligned}
$$

Hence, from Theorem 3, the generator matrix for $C$ is as follows 


$$
\left(\begin{array}{ccccccc}
1 & 1 & 1+u+u^{2} & 1 & 0 & 0 & 0 \\
1 & 1 & 0 & 1+u+u^{2} & 1 & 0 & 0 \\
1 & 1 & 0 & 0 & 1+u+u^{2} & 1 & 0 \\
1 & 1 & 0 & 0 & 0 & 1+u+u^{2} & 1 \\
1 & u & 0 & 0 & 0 & 0 & 0 \\
1 & 1 & u+u^{2} & 0 & u+u^{2} & u+u^{2} & u+u^{2}
\end{array}\right)
$$

Furthermore, the $\Phi(C)$ is a [18, 6, 3] linear binary code.

There is a table about some examples. The binary codes with these parameter are not nice codes. But the binary codes which are marked with * are only about 1 less in minimum distance than the corresponding nice codes.

Table 1. The codes of binary images of $C$.

\begin{tabular}{|c|c|c|}
\hline Generator & $\alpha, \beta, \theta$ & Binary Images \\
\hline $\begin{array}{l}f(x)=1+x, f_{1}(x)=f_{2}(x)=1, g_{1}(x)=1+x, g_{2}(x)=1, \\
a_{1}(x)=1, p(x)=1+x, q(x)=1+x, r(x)=1 .\end{array}$ & $1,1,1$ & {$[6,2,3]^{*}$} \\
\hline$f(x)=1+x, f_{1}(x)=f_{2}(x)=1, g_{1}(x)=1+x^{3}$ & & \\
\hline $\begin{array}{l}g_{2}(x)=1+x+x^{3}, a_{1}(x)=1+x, p(x)=1+x \\
q(x)=1, r(x)=1\end{array}$ & $1,3,1$ & {$[10,3,4]^{*}$} \\
\hline $\begin{array}{l}f(x)=1+x, f_{1}(x)=f_{2}(x)=1, g_{1}(x)=1+x^{3} \\
g_{2}(x)=1+x+x^{3}, a_{1}(x)=1+x, p(x)=1, q(x)=1, r(x)=1\end{array}$ & $1,3,1$ & {$[10,4,3]^{*}$} \\
\hline $\begin{array}{l}f(x)=1+x, f_{1}(x)=f_{2}(x)=1, g_{1}(x)=1+x, g_{2}(x)=1, \\
a_{1}(x)=1, p(x)=1+x, q(x)=1, r(x)=1 .\end{array}$ & $5,1,1$ & {$[10,6,2]^{*}$} \\
\hline $\begin{array}{l}f(x)=1+x, f_{1}(x)=f_{2}(x)=1, g_{1}(x)=1+x^{5}, \\
g_{2}(x)=1+x+x^{2}+x^{3}+x^{4}, a_{1}(x)=1+x+x^{2}+x^{3}+x^{4}, \\
p(x)=1+x, q(x)=1, r(x)=1 .\end{array}$ & $1,5,1$ & {$[14,2,8]^{*}$} \\
\hline $\begin{array}{l}f(x)=1+x, f_{1}(x)=f_{2}(x)=1, g_{1}(x)=1+x^{3}, g_{2}(x)=1+x \\
a_{1}(x)=1+x+x^{2}, p(x)=1+x^{3}, q(x)=1+x, r(x)=1\end{array}$ & $1,3,3$ & {$[16,4,6]$} \\
\hline $\begin{array}{l}f(x)=1+x, f_{1}(x)=f_{2}(x)=1, g_{1}(x)=1+x+x^{2}+x^{3}+x^{4} \\
g_{2}(x)=1+x+x^{2}+x^{3}, a_{1}(x)=1+x+x^{2}+x^{3} \\
p(x)=1+x, q(x)=1+x, r(x)=1\end{array}$ & $1,7,1$ & {$[18,5,6]$} \\
\hline $\begin{array}{l}f(x)=1+x, f_{1}(x)=1, f_{2}(x)=0, g_{1}(x)=1+x^{7}, \\
g_{2}(x)=1+x+x^{2}+x^{3} \\
+x^{4}+x^{5}+x^{6}, \\
q(x)=1, r(x)=1 .\end{array}$ & $1,7,1$ & {$[18,2,10]$} \\
\hline $\begin{array}{l}f(x)=1+x, f_{1}(x)=f_{2}(x)=1, g_{1}(x)=1+x+x^{2}+x^{4}, \\
g_{2}(x)=1+x^{2}+x^{3}, a_{1}(x)=1+x, p(x)=1+x, \\
q(x)=1+x, r(x)=1 .\end{array}$ & $1,7,1$ & {$[18,7,4]$} \\
\hline$f(x)=1+x+x^{2}+x^{4}, f_{1}(x)=f_{2}(x)=1+x^{2}+x^{3}$ & & \\
\hline $\begin{array}{l}g_{1}(x)=1+x^{3}, g_{2}(x)=1+x, a_{1}(x)=1+x+x^{2}, p(x)=1+x^{3}, \\
q(x)=1+x, r(x)=1\end{array}$ & $7,3,3$ & {$[22,7,4]$} \\
\hline
\end{tabular}

\section{Conclusion}

In this paper, we have studied additive cyclic codes over the ring $Z_{2} R S$, where $R=Z_{2}+u Z_{2}, u^{2}=0 \bmod 2$, and $S=Z_{2}+u Z_{2}+u^{2} Z_{2}, u^{2}=0 \bmod 2$. We have given the definition of $Z_{2}\left(Z_{2}+u Z_{2}\right)\left(Z_{2}+u Z_{2}+u^{2} Z_{2}\right)$-additive codes with generator matrices and parity-check matrices. Furthermore, the fundamental results on the generator polynomials and spanning sets for these additive cyclic codes of block length $(\alpha, \beta, \theta)$, where $\theta$ is odd have been obtained. Finding $Z_{2} R S$-additive cyclic code and its dual of 
arbitrary block length $(\alpha, \beta, \theta)$ may be an interesting problem.

\section{References}

[1] Hammons A. R., Kumar P. V., Calderbank A. R., Sloane N. J. A., Sol P., The $Z^{4}$-linearity of Kerdock, Preparata, Goethals and related codes. IEEE Transactions on Information Theory. 40 (2), 301-319, 1994.

[2] Dougherty S. T., Fernndez-Crdoba C., Codes over $Z_{2}{ }^{k}$, Gray Map Self-Dual Codes Adv. Math. Commun. 5 (4), 571-588, 2011.

[3] Greferath M., Schmidt S. E., Gray isometries for finite chain rings and a nonlinear ternary $(36,3 /$ sup $12 /, 15)$ code, IEEE Transactions on Information Theory. 45 (7), 2522-2524, 1999.

[4] Honold T., Landjev I., Linear Codes over Finite Chain Rings, JOURNAL OF COMBINATORICS. 7 (1), R11-R11, 2001.

[5] Delsarte P., Levenshtein V. I., Association schemes and coding theory, IEEE Transactions on Information Theory. 44 (6), 2477-2504, 1998.

[6] Borges J., Fernndez-Crdoba C., Pujol J., Rif J., Villanueva M., $\mathrm{Z}_{2} \mathrm{Z}_{4}$-Linear codes: generator matrices and duality. Designs, Codes Cryptogr. 54 (2), 167-179, 2010.
[7] Abualrub T., Siap I. and Aydin N., $Z_{2} Z_{4}$-Additive cyclic codes, IEEE Transactions on Information Theory. 60 (3), 1508-1514, 2014.

[8] Aydogudu I., Abualrub T. and Siap I., On $\mathrm{Z}_{2} \mathrm{Z}_{2}[\mathrm{u}]$-additive codes, Int. J. Comput. Math. 92 (9), 1806C-1814, 2015.

[9] Borges J., Fernndez-Crdoba C., Ten-Valls R., $Z_{2} Z_{4}$-Additive cyclic codes, generator polynomials and dual codes, IEEE Transactions on Information Theory. 62 (11), 6348-6354, 2016.

[10] Aydogdu I., Gursoy F., $Z_{2} Z_{4} Z_{8}$-Cyclic codes, Journal of Applied Mathematics andm Computing. 60 (1-2), 327-341, 2019.

[11] Joaquim B., Dougherty S. T., Cristina F. C., et. al., Binary Images of $\mathrm{Z}_{2} \mathrm{Z}_{4}$-Additive cyclic codes, IEEE Transactions on Information Theory. 64 (12), 7551-7556, 2018.

[12] Ismail A., Taher A., The structure of $\mathrm{Z}_{2} \mathrm{Z}_{2}{ }^{\mathrm{s}}$-additive cyclic codes, Discrete Mathematics, Algorithms and Applications. 10 (04), 1850048, 2018.

[13] Wan Z. X., Quaternary codes, World Scientific. 8, 1997.

[14] Abualrub T. and Siap I., Cyclic codes over the rings $Z_{2}+u Z_{2}$ and $Z_{2}+u Z_{2}+u^{2} Z_{2}$, Designs Codes and Cryptography. 42 (3), 273-287, 2007.

[15] Macwilliams F. J., Sloane N. J. A., The theory of errorcorrecting codes, Elsevier. 16, 1977. 